\title{
En torno a las políticas sociales Una introducción al Dossier "Políticas sociales y nuevas sociabilidades".
}

\section{About social policies. An introduction to the Dossier "Social policies and new sociabilities"}

\author{
Angélica de Sena*
}

En las economías capitalistas, las políticas sociales se diseñan con el objeto de lograr la integración de la sociedad y la mitigación de las desigualdades propias de las relaciones de producción. Desde esta perspectiva, el proceso social y económico genera un nivel básico de desigualdad y por ello es evidente la necesidad de generar intervenciones por parte de los Estados para compensar los efectos de dichos procesos. Por ello, en las sociedades capitalistas, el problema de la integración social se ubica en el centro de las discusiones respecto al orden social (Grassi, 2006; Castel, 1997, entre otros) y frente a la incapacidad del sistema para asegurar la reproducción de cada una de las personas, es necesario reparar esa falla, y para ello se requiere de la aplicación de políticas sociales con diversos grados de 'complejidad', que acompañen y potencien dicho sistema. De esta manera, las políticas sociales resultan eficaces para menguar la lucha de clases y el conflicto social (Halperín Weisburd, et al., 2008).

Desde hace algunas décadas en América Latina y por tanto en Argentina, se observan y sufren los efectos palpables y dolorosos de los procesos económicos y políticos, dejando claras evidencias del quiebre del derecho a la salud, vivienda, empleo, seguridad social. Se consolida, en consecuencia, un sector social de personas excluidas a quienes, en la última década, se destinaron las políticas sociales de carácter focalizado y masivo

\footnotetext{
*Universidad de Buenos Aires. Correo electrónico: angelicadesena@gmail.com
} 
(De Sena, 2011) con cierto color de asistencialismo, como modo de soportar y hasta ocluir la situación de origen.

Así, y como resultado de las distintas crisis económicas y los distintos ajustes que formaron parte de la matriz neoliberal y su impacto en tanto esquema ideológico y pragmático de un modelo de exclusión, comenzaron a hacerse notar con más fuerza las políticas sociales destinadas a 'suturar'/'crear' las situaciones asociadas al desempleo y caída de los ingresos causados por las denominadas perturbaciones macroeconómicas. De este modo, se evidencia cómo la cuestión del trabajo se funde con la cuestión social.

Las políticas sociales son modos de intervención del Estado que impactan de diversos modos en el tejido social y la vida cotidiana de todas las personas tanto por acción como por omisión; la articulación que los distintos gobiernos $-y$ sus equipos técnicos- establecen entre el reconocimiento de la 'cuestión social', su trasposición en problemas y su intervención contribuyen a establecer su naturaleza, intensidad y límites.

El punto de partida para Danani es que las políticas sociales hacen sociedad, ya que son "aquellas específicas intervenciones sociales del Estado que se orientan (en el sentido de que producen y moldean) directamente a las condiciones de vida y de reproducción de la vida de distintos sectores y grupos sociales, y que lo hacen operando especialmente en el momento de la distribución secundaria del ingreso. En este último aspecto, ello significa que lo que las distingue es que ese proceso de configuración no obra en el circuito de la distribución del ingreso directamente derivada del proceso de producción, por la vía de la retribución a los factores (distribución primaria), sino por mecanismos de redistribución que se le superponen (o, antes bien, sólo analíticamente distinguibles de aquélla, pero tampoco idénticos)" (Danani, 2004: 11).

Dicha definición da cuenta de una amplitud que nos permite buscar especificidades y ampliar miradas y perspectivas teóricas, epistémicas, inter y transdisciplinarias, y nos invita a revisar los procesos y momentos de la política social. 
Danani propone que "el primer vector de diferenciación entre tipos de política es el del objeto sobre el cual ellas actúan directamente (...) lo que es destinatario de la acción u operación del sujeto" (Danani, 2009: 29). Sojo (1990) parte de considerar que la política económica y la social son dimensiones del desarrollo, y afirma que la distribución primaria del ingreso o el empleo son dimensiones propias de la política económica, pero con grandes efectos sociales, por ello estas determinan las políticas sociales.

En un sentido similar, Garretón afirma que "si la finalidad de la política económica es dirigir la economía hacia la satisfacción de necesidades materiales de los individuos (...) la política social tiene por finalidad la producción de las condiciones que aseguran la existencia de la sociedad como tal. Ello significa un cierto nivel de igualdad entre sus miembros, una calidad de vida definida de acuerdo a la diversidad cultural de quienes la forman y la existencia y desarrollo de actores y redes sociales que le den sustento a la ciudadanía" (Garretón, 1999: 42). Por su parte, Grassi (2000) sostiene que hay dos elementos que hacen a la cuestión social en el capitalismo, y que interpelan la legitimidad del Estado moderno democrático. Ellos son: la cuestión de la pobreza, en tanto refiere a las condiciones de reproducción de la vida; es decir, a las limitaciones en el acceso a bienes y servicios y en la participación e integración sociocultural; y a la cuestión del trabajo, entendido como capacidad humana que, organizada por el mercado, viabiliza y legitima el acceso a los recursos, la protección y la seguridad de los sujetos y las condiciones de su participación en la vida sociocultural. Por otro lado, en los últimos años se ha instalado en los debates académicos y políticos el enfoque de derechos humanos, tanto en términos de análisis e implementación de políticas públicas, en vinculación con los modelos de desarrollo, como en relación con las esferas de exigibilidad de los derechos. Este enfoque considera que el marco conceptual que brindan los derechos humanos como derechos legitimados por la comunidad internacional ofrece un sistema coherente de principios y pautas aplicables en las políticas de desarrollo y al mismo tiempo precisa las obligaciones de los Estados en materia de cumplimiento de derechos económicos, sociales y culturales (Abramovich y Pautassi, 2009).

Desde otra vertiente, se pueden pensar las políticas sociales desde la perspectiva de la construcción de la subjetividad, es decir, considerando la 
incidencia que ellas poseen en la producción de sujetos. De ese modo se construye y consolida una subjetividad de 'beneficiario', 'asistido' de la que no es posible salir generando dudas respecto a la efectiva inclusión social en tanto posibilitan la atomización y fragmentación de la experiencia que propician (Pérez Rubio, 2015).

Las políticas sociales son políticas y por ello poseen determinadas concepciones acerca de la problemática que están abordando y de las soluciones acordes, en términos teóricos, metodológicos y epistémicos. Desde esta perspectiva, producen y reproducen procesos que exceden lo meramente material, implicando determinados impactos en la conformación de las subjetividades de las y los actores, configurando y consolidando sensibilidades sociales. El Estado en el diseño e implementación de las políticas sociales establece clasificaciones y categorizaciones sociales, que, como en una correa de transmisión, conforma ideas, valoraciones, formas de sentir y vivencialidades en tanto "manera de expresar los sentidos que adquiere el estar-en-cuerpo con otros como resultado del 'experienciar' la dialéctica entre cuerpo individuo, social y subjetivo, por un lado; y las lógicas de apropiación de las energías corporales y sociales" (Scribano, 2010: 174).

En este breve recorrido hemos intentado mostrar algunos de los posibles modos de revisar las políticas sociales con el objeto de presentar los artículos que conforman el Dossier de este número de la revista De Prácticas y Discursos, abriendo con una reflexión de carácter histórico: El Certificado Médico Prenupcial como política social (1936-1955) de Valeria Almirón y Carolina Biernat. Las autoras revisan el proceso de implementación de la normativa argentina en el año 1936, respecto a la obligatoriedad del certificado prenupcial para los varones como respuesta a las demandas de círculos profesionales, académicos y políticos en torno a la necesidad de dar respuesta a la escalada de los índices de morbilidad venérea.

Este recorrido sigue con un artículo de Ana Laura HIDALGO, en donde las políticas sociales también se relacionan con el trabajo: Economía social e ingresos. Algunas tensiones en su articulación en la implementación del Programa de Microcrédito en la ciudad de San Luis. El artículo se propone 
poner en relación las experiencias de la Economía Social y Solidaria y el porcentaje de los ingresos que los sujetos perciben de su actividad emprendedora, a fin de reconocer las tensiones entre ambas.

En otra línea, Gabriela Vergara y Pedro Lisdero nos proponen revisar la basura y el conflicto como dos conceptos unidos por la pobreza. Gestionar, protestar y vivir de la basura. Un análisis desde el conflicto social en torno a las políticas públicas sobre los RSU en Córdoba analiza las protestas y vivencias de recuperadores de residuos como una vía para problematizar las relaciones entre políticas públicas, sensibilidades y procesos de estructuración social.

El siguiente artículo Apuntes para la problematización: ¿desmercantilización o re-mercantilización de la política social actual?: Transferencias Estatales y consumo como aspectos a considerar, de Andrea Dettano, busca articular la literatura sobre las esferas de la provisión de bienestar en pos de hacer una lectura focalizada sobre la política social actual y la esfera de la desmercantilización. Ello para revisar el potencial mercantilizador/desmercantilizador de la Asignación Universal por Hijo para la Protección social, que funciona en Argentina desde 2009 y su incentivo al consumo.

Los siguientes dos artículos miran la Ciudad Autónoma de Buenos Aires, Melina Patti a través de Una descripción y caracterización del Programa de Transferencias Condicionadas “Ciudadanía Porteña-Con todo derecho, se propone contribuir a una caracterización detenida de dicho programa haciendo énfasis en el relevamiento de aspectos vinculados a sus contraprestaciones en salud y educación.

En esta línea temática, Ángeles Bermúdez, Verónica Carmona Barrenechea, Giuseppe Messina y Laura Royo presentan el artículo El derecho a la alimentación en la Ciudad de Buenos Aires, una mirada desde las políticas públicas, en el que abordan la cuestión alimentaria a través del análisis de los programas Ticket Social y Ciudadanía Porteña.

Y como cierre y continuidad con la cuestión alimentaria, por un lado, pero también de políticas al respecto, Maximiliano Carrasco y Laura Pautassi 
analizan Diez años del "Plan Nacional de Seguridad Alimentaria" en Argentina. Una aproximación desde el enfoque de derechos en tanto metodología que establece las vinculaciones entre las obligaciones contenidas en Pactos y Tratados Internacionales de Derechos Humanos y su incorporación en las políticas públicas.

Desde estos lugares, el presente número de De Practicas y Discursos se involucra con la morfología de las políticas sociales, revisando intervenciones específicas del Estado que configuran un tejido social que traman estructuras de subjetividades y sensibilidades y organizan la vida de millones de personas. Invitamos a los lectores no solo a la lectura del número, sino a reflexionar en torno a las complejidades de lo social. El presente artículo tiene como objetivo presentar una mirada, a la luz del enfoque de los derechos humanos, de la principal política alimentaria implementada en el país postcrisis del año 2001/02: el "Plan Nacional de Seguridad Alimentaria" (PNSA). Partimos por definir qué se entiende por enfoque de derechos, precisando su aplicación para garantizar el Derecho Humano a la Alimentación Adecuada (DaAA), para, una vez introducidas las definiciones, realizar un análisis del plan en base a documentos oficiales de los poderes Ejecutivo y Legislativo nacionales y de organismos de control nacionales. Para ello, relevamos los informes de la Auditoría General de la Nación, la evolución presupuestaria del PNSA desde el año 2003 al año 2012 en base a datos obtenidos del Ministerio de Economía de la Nación y la totalidad de iniciativas legislativas presentadas en el Congreso Nacional referidas al PNSA entre 2002 y agosto de 2013. ${ }^{1}$ Sobre esta base empírica, las conclusiones recuperan aspectos críticos del programa, que implican limitaciones para garantizar el Derecho a la Alimentación Adecuada, formulando propuestas de abordaje superadoras de los límites identificados.

\section{Bibliografía}

- Abramovich, V. y Pautassi, L. (2009). El enfoque de derechos y la institucionalidad de las políticas sociales. En Abramovich, V. y Pautassi, L.

\footnotetext{
${ }^{1}$ La actividad de relevamiento se realizó entre los meses de abril y agosto de 2013 en el marco del proyecto "Políticas sociales y legislación para la garantía y protección del Derecho a la Alimentación Adecuada en Argentina", seleccionado en la II Convocatoria de Investigaciones del Observatorio del Derecho a la Alimentación en América Latina-FAO, http://www.oda-alc.org/
} 
(Comp.) La revisión judicial de las políticas sociales. Buenos Aires: Editores del Puerto.

- Castel, R. (1997). Las metamorfosis de la cuestión social. Una crónica del Salariado. Buenos Aires: Ed. Paidós.

- De Sena, A. (2011). Promoción de Microemprendimientos y políticas sociales: ¿Universalidad, Focalización o Masividad?, una discusión no acabada. En Revista Pensamento Plural. Instituto de Sociologia e Política. Mestrado em Ciências Sociais. Universidade Federal de Pelotas. Año 4 Número 8 Enero-Junio 2011 (Pp. 36-66) Pelotas, Brasil.

- De Sena, A. y Cena, R. (2014). ¿Qué son las políticas sociales? Esbozos de respuestas. En De Sena, A. (Ed.) Las políticas hechas cuerpo y lo social devenido emoción: lecturas sociológicas de las políticas sociales. Estudios Sociológicos Editora/Universitas. Editorial Científica Universitaria. Argentina. (Pp.19-50). Disponible en: http://estudiosociologicos.org/portal/lecturassociologicas-de-las-politicas/

- Danani, C. (2004). El alfiler en la silla: sentidos, proyectos y alternativas en el debate de las políticas sociales y de la economía social. En Danani, C. (Comp.) Política social y economía social. Universidad Nacional de General Sarmiento-Fundación OSDE. Buenos Aires: Ed. Altamira.

(2009). La gestión de la política social: un intento de aportar a su problematización. En Chiara, M. y Di Virgilio, M. (Orgs.) Gestión de la política social. Conceptos y herramientas. Universidad Nacional de General Sarmiento. Buenos Aires: Ed. Prometeo.

- Garretón, M. (1999). Igualdad, ciudadanía y actores en las políticas sociales. Revista de Ciencias Sociales. Iquique. Chile. № 009. Universidad Arturo Prat.

- Grassi, E. (2006). Integración y necesidades sociales. Reflexiones desde el punto de vista de la igualdad. Ponencia presentada en el Tercer Congreso Argentino de Política Social. Buenos Aires.

- Grassi, E. (2000). Procesos Político-culturales en torno del trabajo. Acerca de la problematización de la cuestión social en la década de los 90 y el sentido de las "soluciones" propuestas: un repaso para pensar el futuro. Revista Sociedad N ${ }^{\circ} 16$ Facultad de Ciencias Sociales, UBA. Buenos Aires. Universidad de Buenos Aires.

- Halperin Weisburd, L. Et al (2008). Políticas sociales en la Argentina. Entre la ciudadanía plena y el asistencialismo focalizado en la contención del pauperismo. Documento de Trabajo No 13. Centro de Estudios sobre Población, Empleo y Desarrollo (CEPED). Facultad de Ciencias Económicas. Universidad de Buenos Aires. Buenos Aires.

- Hopp, M. (2010). Trabajo e integración social en las políticas de desarrollo socio-productivo en la Argentina (2003-2008). Documento de jóvenes investigadores $\mathrm{N}^{\circ} 25$. Instituto de Investigaciones Gino Germani. Facultad de Ciencias Sociales. Universidad de Buenos Aires. Buenos Aires.

Scribano, A. (2007). La Sociedad hecha callo: conflictividad, dolor social y regulación de las sensaciones (pp. 118-142). En Scribano, A. (Comp.) 
Mapeando Interiores. Cuerpo, Conflicto y Sensaciones. CEA-UNC.. Córdoba, Argentina: Jorge Sarmiento Editor.

Pérez Rubio, A. M. (2015). Las políticas sociales como instituciones totales: la construcción de la subjetividad. En Pérez Rubio, A. M., Barbetti, P. (Coords.) Políticas Sociales: significaciones y prácticas. ESEditora (en prensa).

Sojo, A. (2007). La trayectoria del vínculo entre políticas selectivas contra la pobreza y políticas sectoriales. Revista de la CEPAL. Santiago de Chile. N. ${ }^{\text {ro }}$ 91. Abril 2007. 